\title{
Lateral Ventricle Segmentation of 3D Pre-term Neonates US Using Convex Optimization
}

\author{
Wu Qiu, Jing Yuan, Jessica Kishimoto, Eranga Ukwatta, and Aaron Fenster \\ Robarts Research Institute, University of Western Ontario, London, ON, Canada
}

\begin{abstract}
Intraventricular hemorrhage (IVH) is a common disease among preterm infants with an occurrence of $12-20 \%$ in those born at less than 35 weeks gestational age. Neonates at risk of IVH are monitored by conventional 2D ultrasound (US) for hemorrhage and potential ventricular dilation. Compared to 2D US relying on linear measurements from a single slice and visually estimates to determine ventricular dilation, 3D US can provide volumetric ventricle measurements, more sensitive to longitudinal changes in ventricular volume. In this work, we propose a global optimization-based surface evolution approach to the segmentation of the lateral ventricles in preterm neonates with IVH. The proposed segmentation approach makes use of convex optimization technique in combination with a subject-specific shape model. We show that the introduced challenging combinatorial optimization problem can be solved globally by means of convex relaxation. In this regard, we propose a coupled continuous max-flow model, which derives a new and efficient dual based algorithm, that can be implemented on GPUs to achieve a highperformance in numerics. Experiments demonstrate the advantages of our approach in both accuracy and efficiency. To the best of our knowledge, this paper reports the first study on semi-automatic segmentation of lateral ventricles in neonates with IVH from 3D US images.
\end{abstract}

Keywords: lateral ventricle segmentation, convex optimization, pre-term neonate, 3D ultrasound imaging.

\section{Introduction}

Preterm neonates that are born of low birth weight $(<1500 \mathrm{~g})$ are at an increased risk of intraventricular hemorrhage (IVH) [1], bleeding in and around the ventricles. Diagnosis of IVH is done using 2D clinical ultrasound (US). The hemorrhage is ranked using the Papile grading system 2 that qualitatively describes the location and approximate volume of ventricle affected. By this system, grade I and II indicate a minor hemorrhage and tends towards favourable patient outcomes, and grades III and IV indicate severe hemorrhage often with following ventricular dilation (ventriculomegaly) and poor patient outcomes. Patients often have ventriculomegaly that spontaneously resolves, but some fraction of those will progress to hydrocephalus, and may require interventional therapy to reduce the amount of cerebral spinal fluid (CSF) accumulated in the brain. 3D US can monitor the ventricular system in neonates [34, and can be done at the 
bed side, however; to incorporate 3D US into clinical setting, a segmentation algorithm would be required to adequately reduce the time required to obtain the ventricle volume from the 3D image.

Previous cerebral ventricle segmentation algorithms have been exclusively for CT [5] and MRI images [6], and mainly for adult populations. While studies have quantified 3D US ventricle volumes in neonates [34], all have used expert manually contoured regions in lieu of a semi-automatic or automatic approach. Unlike in a healthy neonate, IVH patients ventricles provide an increased difficultly to segment due to both the bleed itself as well as the irregular manner in which the ventricle deforms. The bleeding creates a hyperechoic region around and inside the relatively hypoechoic region of the CSF filled ventricles making most threshold or boundary based segmentation algorithms not useful for this application. Atlas based segmentations fail as dilation and subsequent reduction in conjunction with brain growth cause deformations to the structure that cannot be accounted for and vary drastically from patient to patient. Manual segmentation, though done in previous studies to quantify 3D US ventricle volumes [34], is too arduous and time consuming to be clinically feasible. Moreover, manual segmentation of such a structure is challenging due to indistinct boundaries and irregular shape deformation, even for an expert. Thus, accurate and efficient automatic or semi-automatic ventricle segmentation is highly desired in clinical practice.

Contributions: In this study, we propose convex optimization based approach for delineating lateral ventricles of pre-term neonates from 3D US images, which incorporates a subject-specific model as the shape prior. We show that the introduced challenging combinatorial optimization problem can be globally optimized by means of convex relaxation. Moreover, we introduce a coupled continuous max-flow model which is equivalent to the formulated convex relaxed optimization problem. With the help of the coupled continuous max-flow formulation, we derive an efficient multiplier augmented algorithm, which avoid directly tackling the original non-smooth convex energy functional and can be readily implemented on a GPU to achieve a substantial speed-up in computation. To the best of our knowledge, this paper reports the first study on semi-automatic segmentation of lateral ventricles of premature newborn brains from 3D US images.

\section{Method}

\subsection{Segmentation Pipeline}

A specific subject model is used to facilitate the segmentation task considering great individual shape variations and inhomogeneous context. A 3D US image at baseline is first segmented manually by an expert. Then, two segmented lateral ventricle surfaces (left and right lateral ventricles) as subject-specific models are rigidly registered to the following repeat images (from the second time-point) through six chosen landmarks. The registered models are used as an initial guess of lateral ventricles for repeat images, estimating the prior probability density 


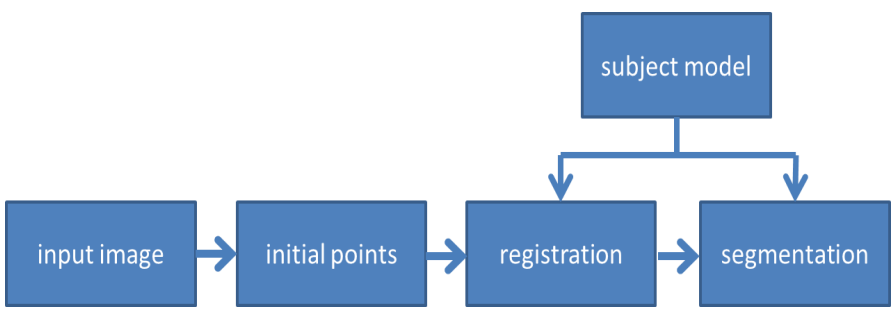

(a) segmentation pipeline

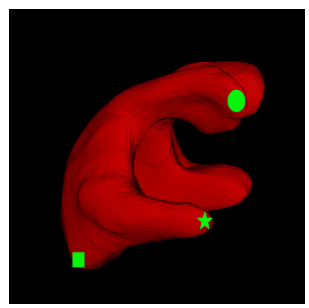

(b) subject model

Fig. 1. Schematic diagram of segmentation pipeline; (a) segmentation pipeline, (b) example of a 3D rendered ventricle model with landmarks

functions (PDF) for the background and foreground. The subject-specified models also served as shape priors to assist the subsequent convex optimization based segmentation algorithm. Figure 1(a) shows the segmentation pipeline.

\subsection{Rigid Registration}

Three landmarks, locating at anterior, posterior, and inferior horn of right lateral ventricle, respectively (green circle, square, and star in Fig. 1(b)), are manually chosen from each ventricle in a given subject-specified model at baseline, resulting in six landmarks for the two ventricles. Six corresponding initial landmarks are also manually chosen from the repeat images. An affine transformation is then calculated from those six corresponding point pairs, which are used to rigidly register the subject model to the following repeat images. It should be noted that a rigid registration can not guarantee a good segmentation for repeat images due to the nonlinear deformation caused by neonate brain growing, ventricle deformation and bleeding. Thus, a more accurate segmentation algorithm is needed to refine the rough registration initial result.

\subsection{Convex Optimization Based Surface Evolution with Shape Constraint}

In this work, a convex optimization based method with shape constraint was used to refine the registered surface. We denote $\mathcal{R}_{i}, i=L, R$, and $B$ as the left ventricle, right ventricle, and background pixels, respectively; $u_{i} \in\{0,1\}$, $i=L, R$, and $B$ are denoted as the corresponding indicator labeling functions of $\mathcal{R}_{L}, \mathcal{R}_{R}$, and $\mathcal{R}_{B}$, respectively. Since there is no overlap between left and right ventricle in $\mathcal{R}$, we have

$$
u_{L} \cap u_{R}=\varnothing, \quad \forall x \in \mathcal{R} .
$$

Multiple-Region Segmentation: The segmentation of $\mathcal{R}$ into three regions of $\mathcal{R}_{B}, \mathcal{R}_{L}$ and $\mathcal{R}_{R}$ can be formulated as a coupled continuous min-cut problem, which minimizes the following energy function:

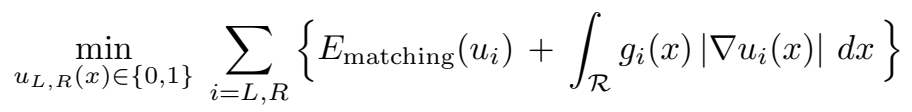


where $E_{\text {matching }}(u)$ is Bhattacharyya distance [7/8] used to measure the distance between the estimated PDFs for the two estimated regions and their corresponding PDF models(see Section 2.1); the weight function $g(x)$ in (2) is positive and is given by

$$
g(x)=\lambda_{1}+\lambda_{2} \exp \left(-\lambda_{3}|\nabla I(x)|\right), \quad \lambda_{1,2,3} \geq 0 .
$$

Note that, $I(x) \in \mathcal{Z}$ be a given repeat image, where $\mathcal{Z}$ is the set of image intensity values, and the values of $g(x)$ fall within the range $\left[\lambda_{1}, \lambda_{1}+\lambda_{2}\right]$. We can estimate the PDF $p_{i}(u, z)$, where $z \in \mathcal{Z}$ and $i=L, R, B$, for the estimated region $\mathcal{R}$ by the Parzen method [9], such that

$$
p(u, z)=\frac{\int_{\mathcal{R}} K(z-I(x)) u d x}{\int u d x},
$$

where $K(\cdot)$ is the Gaussian kernel function such that $K(x)=\frac{1}{\sqrt{2 \pi \sigma^{2}}} \exp \left(-x^{2} / 2 \sigma^{2}\right)$. $q(z)$ is defined as the intensity $\mathrm{PDF}$ model of the region $\mathcal{R}$, where $z \in \mathcal{Z}$. Thus, $E_{\text {matching }}(u)$ is defined as:

$$
E_{\text {matching }}(u)=-\sum_{z \in \mathcal{Z}} \sqrt{p(u, z) q(z)}
$$

Convex optimization with shape constraint: In addition, we penalize the difference between the evolved ventricle surface and the registered subject-specified model so as to enforce that the resulting surface is not far from the initial guess. Let $\mathcal{C}^{*}$ be the registered surface model, and $u^{*}(x) \in\{0,1\}$ the indicator function of $\mathcal{C}^{*}$, which is used to assist the segmentation task of $\mathcal{C}$ [10. The indicator function $u(x) \in\{0,1\}$ of the ventricle regions within $\mathcal{C}$ is optimized over the following energy function:

$\min _{u_{L, R}(x) \in\{0,1\}} \sum_{i=L, R}\left\{E_{\text {matching }}\left(u_{i}\right)+\omega_{1} \int_{\mathcal{R}} g_{i}(x)\left|\nabla u_{i}(x)\right| d x+\omega_{2} \int_{\mathcal{R}}\left|u_{i}-u_{i}^{*}\right| d x\right\}$

where $E_{\text {matching }}(u)$ formulates the statistical intensity distribution matching energy inside and outside two ventricles, the second weighted total-variation function gives the surface smoothness term and $\omega_{1,2}>0$ are the positive penalty parameters $\left(\omega_{1}=0.3\right.$ and $\omega_{2}=0.05$ were used in our experiments). The last energy term of (5) encodes and penalizes the difference between the evolved ventricle surface and the registered model.

To optimize the energy function (5), which is often highly nonlinear, we introduce the convex optimization based evolution approach [11, which can efficiently move the given surface to the object of interest. In contrast to the traditional evolution methods, e.g. active contour or level-set, the introduced surface evolution approach provides a fully time implicit scheme in numerics, for which a large time step-size is allowed to significantly speed up the surface evolution process and, during each discrete evolution time frame, the surface can be moved to its 
globally optimal position by globally solving the following continuous min-cut problem:

$$
\min _{u_{L, R}(x) \in\{0,1\}} \sum_{i=L, R}\left\{\left\langle 1-u_{i}, C_{s}^{i}\right\rangle+\left\langle u_{i}, C_{t}^{i}\right\rangle+\int_{\mathcal{R}} g_{i}(x)\left|\nabla u_{i}\right| d x\right\}
$$

where the functions $C_{s, t}^{i}(x)$ are set up w.r.t. the current surface[11. The continuous min-cut problem (6) can be solved globally and efficiently by means of the continuous max-flow method 12 and implemented on the modern parallel computing platforms (GPUs) to obtain the high-performance in numerics. The proof of the duality between the continuous max-flow model and the convex relaxed optimization model (6) is omitted due to the limited space.

\section{$3 \quad$ Experiments}

Image acquisition: A motorized 3D US system developed for cranial US scanning of pre-term neonates was used to acquire the images. Following the routine cranial US exam, the 2D US transducer (Phillips C8-5 broadband curved array) is placed into the motorized housing and the 3D US image is acquired. The US technician locates the midline of lateral ventricles through the anterior fontanelle. Images are then acquired while the motor housing is held firmly while the device rotates the transducer at an axis at the probe tip. Scans were performed with a 60-72 degree scan angle, a step size of 0.3 degrees at a frame rate of 25 frames/s with scan times between 8-12 seconds 13 . The image sizes ranged from $300 \times 300 \times 300$ to $450 \times 450 \times 450$ voxels at the same voxel spacing of $0.22 \times 0.22 \times 0.22 \mathrm{~mm}^{3}$. Scans were performed 1-2 times per week for the first month enrolled in the study and 1-4 per month for the duration of the patients stay in the neonatal intensive care unit.

Evaluation Metrics: Ventricles were manually segmented in parallel slices of the 3D US image with $0.75 \mathrm{~mm}$ between slices. Our segmentation method was then evaluated by comparing the algorithm to manual segmentation results using volume-based metrics: Dice similarity coefficient (DSC) 14]; and distance-based metrics: the mean absolute surface distance (MAD) and maximum absolute surface distance (MAXD) [15]. In addition, each image was segmented three times by the same observer for assessing the intra-observer variability. The mean run time of three repeated segmentations for each 3D US image was considered as the segmentation time to assess the algorithm's efficiency.

\section{Results}

Accuracy: Figure. 2 shows two algorithm segmented lateral ventricles (green contours) and manual delineations (red contours) of one patient, demonstrating good agreement. Table. 1 shows the mean quantitative segmentation results for 20 patient images using the proposed method. Our approach obtained a mean 


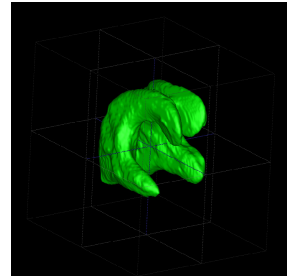

(a) segmented surface

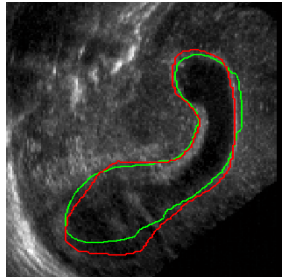

(b) sagittal view

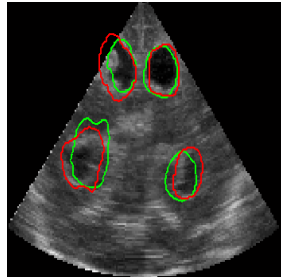

(c) coronal view

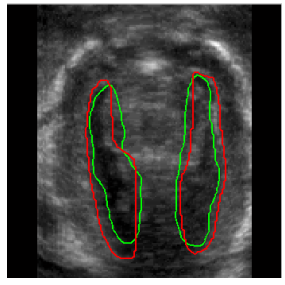

(d) transvers view

Fig. 2. Segmented ventricles (green contour) overlapped with manual segmentations (red contour)

Table 1. Segmentation results of twenty 3D US images of four patients (five time points each) in terms of DSC, MAD, and MAXD (p1-p4: patient ID)

\begin{tabular}{cccccc}
\hline & $\mathrm{p} 1$ & $\mathrm{p} 2$ & $\mathrm{p} 3$ & $\mathrm{p} 4$ & Mean \\
\hline DSC (\%) & $71.2 \pm 1.5$ & $74.3 \pm 2.0$ & $70.5 \pm 2.1$ & $70.5 \pm 2.1$ & $\mathbf{7 2 . 4} \pm \mathbf{2 . 5}$ \\
MAD (mm) & $0.5 \pm 0.2$ & $0.6 \pm 0.1$ & $0.7 \pm 0.1$ & $0.7 \pm 0.1$ & $\mathbf{0 . 7} \pm \mathbf{0 . 1}$ \\
MAXD (mm) & $3.8 \pm 1.2$ & $2.7 \pm 1.6$ & $3.1 \pm 2.2$ & $3.4 \pm 1.5$ & $\mathbf{3 . 5} \pm \mathbf{1 . 6}$ \\
\hline
\end{tabular}

DSC of $72.4 \pm 2.5 \%$, a MAD of $0.7 \pm 0.1 \mathrm{~mm}$, and a MAXD of $3.5 \pm 1.6 \mathrm{~mm}$ for the patients' two ventricles. Considering that values of DSC above $70 \%$ are usually regarded as a satisfactory level of agreement between two segmentations 1416, the segmentation accuracy generated by our method is useful for clinic use.

Reiliability: The result of the intra-observer variability test showed that the proposed method yielded a DSC of $72.5 \pm 2.1 \%, 73.4 \pm 2.6 \%$ and $73.2 \pm 2.4 \%$ for three segmentations from the same observer, respectively. ANOVA analysis with a single factor failed to demonstrate a statistically significant difference between these three segmentations $(p=0.80, F=0.75)$. The approximately similar DSCs yielded by algorithm in all three repetitions suggest a high reproducibility of our approach.

Computational Time: The proposed approach was implemented using parallel computing architecture (CUDA, NVIDIA Corp., Santa Clara, CA) and the user interface in Matlab (Natick, MA). The experiments were conducted on a Windows desktop with an Intel i7-2600 CPU $(3.4 \mathrm{GHz})$ and a GPU of NVIDIA Geforce 5800X. The segmentation time including registration and convex optimization was calculated as the mean run time of three repeated segmentations for each 3D US image. The mean segmentation time of our method was $15 \pm 2 \mathrm{~s}$ in addition to $50 \pm 5 \mathrm{~s}$ for initialization, resulting in a total segmentation time of less than $\mathbf{1 . 5}$ minutes for a single 3D lateral ventricle US image, significantly less than 30 minutes for manual segmentation. 


\section{Discussion and Conclusion}

This paper proposes an accurate, reproducible and numerically efficient solution to a challenging segmentation problem of the lateral ventricles of pre-term neonates from 3D US images, which makes use of the latest development of convex optimization technique combined with a subject-specific shape model. The experimental results show that the proposed method is reliable to the initial segmentation of the first image, where the rough manual segmentation is registered to the other images as the shape prior, in combination with the local image features, to assist the segmentation of the following images. In practice, the local image appearance can derive the correct region boundaries even when the shape prior has errors. In addition, the intra-observer experiments show that the variability introduced by the user-selected shape prior is small in terms of DSC, which demonstrates that the proposed method is reliable at least for a single expert. Although the current algorithm is semi-automatic, and still requires manual segmentation for the first image, the $1.5 \mathrm{~min}$ computational time is still promising for repeat images in clinical trials compared to half an hour for a currently used manual segmentation, The algorithm performance suggests that it has the potential to be used for measuring the volume of lateral ventricles of pre-term neonates.

Many investigators are focusing on segmentation of brain anatomic structures in 3D MRI, such as lateral ventricle and hippocampus. However, these are found to be application-dependent, which can not be directly applied in our 3D US application due to the difference of modality and segmentation object. We tested the classic active contour [17], level set [18], and graph cut [19] algorithms in our dataset. Visual inspection show that all of these failed to generate favourable results for all testing images. It should be noted that our algorithm cannot handle the situation when bleeding extends outside ventricles (grade IV haemorrhage), which greatly concerns obstetricians and neonatologist along with ventricle volume.

Acknowledgments. The authors are grateful for the funding support from the Canadian Institutes of Health Research (CIHR), the Ontario Institute of Cancer Research (OICR), the Canada Research Chairs (CRC) Program, and Academic Medical Organization of Southwestern Ontario (AMOSO).

\section{References}

1. Wilson-Costello, D., Friedman, H., Minich, N., Fanaroff, A.A., Hack, M.: Improved survival rates with increased neurodevelopmental disability for extremely low birth weight infants in the 1990s. Pediatrics 115(4), 997-1003 (2005)

2. Papile, L.A., Burstein, J., Burstein, R., Koffler, H.: Incidence and evolution of subependymal and intraventricular hemorrhage: a study of infants with birth weights less than 1,500 gm. The Journal of Pediatrics 92(4), 529-534 (1978)

3. Abdul-Khaliq, H., Vogel, M., Lange, P.: Feasibility of brain volumetric analysis and reconstruction of images by transfontanel three-dimensional ultrasound. Journal of NeuroImaging 10(3), 147-150 (2000) 
4. McLean, G., Coombs, P., Sehgal, A., Paul, E., Zamani, L., Gilbertson, T., Ptasznik, R.: Measurement of the lateral ventricles in the neonatal head: Comparison of 2-d and 3-d techniques. Ultrasound in Medicine \& Biology (2012)

5. Liu, J., Huang, S., Ihar, V., Ambrosius, W., Lee, L.C., Nowinski, W.L.: Automatic model-guided segmentation of the human brain ventricular system from ct images. Academic Radiology 17(6), 718-726 (2010)

6. Liu, J., Huang, S., Nowinski, W.L.: Automatic segmentation of the human brain ventricles from $\mathrm{mr}$ images by knowledge-based region growing and trimming. Neuroinformatics 7(2), 131-146 (2009)

7. Michailovich, O., Rathi, Y., Tannenbaum, A.: Image segmentation using active contours driven by the bhattacharyya gradient flow. IEEE Transactions on Image Processing 16(11), 2787-2801 (2007)

8. Ukwatta, E., Yuan, J., Rajchl, M., Qiu, W., Tessier, D., Fenster, A.: 3d carotid multi-region MRI segmentation by globally optimal evolution of coupled surfaces. IEEE Tran. Med. Imag. 32(4), 770-785 (2013)

9. Parzen, E.: On estimation of a probability density function and mode. The Annals of Mathematical Statistics 33(3), 1065-1076 (1962)

10. Qiu, W., Yuan, J., Ukwatta, E., Tessier, D., Fenster, A.: Prostate segmentation in $3 \mathrm{~d}$ TURS using convex optimization with shape constraint. In: SPIE, Medical Imaging (2013)

11. Yuan, J., Ukwatta, E., Tai, X.C., Fenster, A., Schnoerr, C.: A fast global optimization-based approach to evolving contours with generic shape prior. Technical report CAM-12-38, UCLA (2012)

12. Yuan, J., Bae, E., Tai, X.: A study on continuous max-flow and min-cut approaches. In: CVPR 2010 (2010)

13. Kishimoto, J., Lee, D., Lawrence, K.S., Romano, W., Fenster, A., de Ribaupierre, S.: Development of a 3d ultrasound system to investigate post-hemorrhagic hydrocephalus in pre-term neonates. In: SPIE Medical Imaging, p. 86751M (2013)

14. Zijdenbos, A.P., Dawant, B.M., Margolin, R.A., Palmer, A.C.: Morphometric analysis of white matter lesions in $\mathrm{mr}$ images: method and validation. IEEE Transactions on Medical Imaging 13(4), 716-724 (1994)

15. Garnier, C., Bellanger, J.J., Wu, K., Shu, H., Costet, N., Mathieu, R., de Crevoisier, R., Coatrieux, J.L.: Prostate segmentation in HIFU therapy. IEEE Trans. Med. Imag. 30(3), 792-803 (2011)

16. Xue, H., Srinivasan, L., Jiang, S., Rutherford, M., Edwards, A.D., Rueckert, D., Hajnal, J.V.: Automatic segmentation and reconstruction of the cortex from neonatal mri. Neuroimage 38(3), 461-477 (2007)

17. Kass, M., Witkin, A., Terzopoulos, D.: Snakes: Active contour models. International Journal of Computer Vision 1(4), 321-331 (1988)

18. Chan, T.F., Vese, L.A.: Active contours without edges. IEEE Trans. Img. Process. 10(2), 266-277 (2001)

19. Boykov, Y., Veksler, O., Zabih, R.: Fast approximate energy minimization via graph cuts. IEEE Transactions on Pattern Analysis and Machine Intelligence 23(11), 1222-1239 (2001) 